\title{
PROPERTIES OF HOT MASSIVE STARS FROM STUDIES OF THEIR RING NEBULAE
}

\author{
LINDA J. SMITH \\ Department of Physics and Astronomy, University College London \\ Gower Street, London WC1E 6BT, U.K.
}

\begin{abstract}
Wolf-Rayet (WR) stars and their possible precursors, the Luminous Blue Variables (LBVs), are often surrounded by ring nebulae. It is believed that these nebulae are formed by the action of the stellar wind, matter being ejected from the star in the past, or a combination of these two processes. The various research applications of LBV and WR nebulae are reviewed with regard to the information they provide on the properties of the central stars. They are very useful probes of stellar evolution since the details of the previous evolutionary phases of the central stars are contained in the nebulae. In particular, abundance studies can provide insights into the chemical processes operating during the precursor phases. The nebulae can also be used as probes of the far-UV ionizing flux distribution of the central WR stars by comparing the observed levels of nebular ionization with those produced with non-LTE WR model flux distributions. Comparisons of stellar effective temperatures and luminosities derived using this technique with those determined by modelling stellar emission lines can identify deficiencies such as the lack of line-blanketing in WR model atmospheres. Very hot WR stars can also be identified by searching for nebular HeII emission. Studies of bipolar structures in LBV and WR nebulae provide valuable clues on wind asymmetries in the central stars.
\end{abstract}

Key words: stars: Wolf-Rayet - Luminous Blue Variables - ring nebulae

\section{Introduction}

The ring-shaped nebulae that surround some Wolf-Rayet (WR) stars, and their possible precursors, the Luminous Blue Variables (LBVs), contain a great deal of information about the central stars. They provide a record of the past mass loss history, whether it is in the form of bulk ejections or stellar wind flows. Abundance analyses can identify nebulae composed of stellar ejecta, and the resulting abundance ratios can be used to probe the past evolutionary history of the central star. Studies of the morphology of LBV and WR ejecta-type nebulae have the potential of providing information on wind asymmetries. Since these nebulae are photoionized by their central stars, effective temperatures and luminosities can be obtained by modelling their observed ionization structures. Overall, one of the main advantages of using ring nebulae as probes of the central stars is that the input physics is relatively simple compared with complex stellar atmosphere analyses. The disadvantage is that the nebulae are faint and large amounts of telescope time are required to obtain spectra covering important weak diagnostic lines for many different slit positions. 
A WR ring nebula is usually defined as a ring or arc of nebulosity centred on and ionized by the central star. The first three such nebulae (NGC 2359, NGC 6888 and S 308) were discovered by Johnson \& Hogg (1965). Many more Galactic WR ring nebulae have since been identified in systematic searches. The atlas of Chu et al. (1983) lists 15 probable and 3 possible Galactic WR nebulae based on photographic surveys (Chu 1981; Heckathorn et al. 1982). More recently, CCD-based surveys have been completed for the northern (Miller \& Chu 1993) and southern hemispheres (Marston et al. 1994a,b) as well as the Large Magellanic Cloud (Dopita et al. 1994). These new surveys have higher sensitivity than the earlier photographic work, resulting in the identification of 3 new probable and 4 possible Galactic WR ring nebulae. For the LMC, a complete survey of the known 115 WR stars was carried out, leading to the discovery of many new small ring nebulae such that $16 \%$ of WR stars in the LMC are surrounded by ring nebulae (Dopita et al. 1994). Ring nebulae have also been discovered and studied around WR stars in M33 (Drissen et al. 1991; Esteban et al. 1994). The diameters and masses of WR nebulae cover a wide range of $2-20 \mathrm{pc}$ and $1-40 \mathrm{M}_{\odot}($ e.g., Chu et al. 1983), suggesting different formation mechanisms.

Regarding other massive stars, some Of and OB stars are associated with nebulae or bow shocks (e.g., Chu 1991). Of particular interest are the nebulae surrounding Luminous Blue Variable stars (LBVs) since they are thought to represent a brief, unstable phase in the evolution of a massive $O$ star to a helium-burning WR star. To date, about ten Galactic LBVs are known of which eight have nebulae (e.g., $\eta$ Car, AG Car, P Cyg, HR Car, WRA 751). The diameters and masses of these LBV nebulae are much lower than those of most WR nebulae with typical values of $0.2-2 \mathrm{pc}$ and $0.1-$ $4 \mathrm{M}_{\odot}$ (Hutsemékers 1994). These small diameters and low masses suggest that LBV nebulae are composed mainly of stellar ejecta, in accord with the historical evidence for large eruptions in $\eta$ Car and P Cyg.

\section{Nebulae as probes of stellar evolution}

\subsection{Formation of WR and LBV nebulae}

The formation and composition of the nebulae surrounding WR stars and LBVs are complex when the evolutionary details of the central stars are considered. Following the standard evolutionary picture (e.g., Chiosi \& Maeder 1986), if the initial mass of the $O$ star is $\sim 25-50 \mathrm{M}_{\odot}$, then evolution first proceeds to the red in the HR diagram, where the hydrogen rich layers are lost in a red supergiant (RSG) phase, before the star turns back to the blue as a WR star. If the $O$ star is more massive than $\sim 50 \mathrm{M}_{\odot}$, then the star remains in the blue, and enters a short-lived LBV phase immediately before the WR stage.

For the formation and subsequent evolution of the nebulae, there are three 
main stages to consider. The first stage is the evacuation of the surrounding interstellar medium (ISM) by the $\mathrm{O}$ star wind to form a wind-blown bubble (WBB). The second, and most important stage for evolutionary studies, is either the interaction of the denser wind of the RSG with the ISM, or the interaction of the wind plus bulk ejections from the unstable LBV phase. The third stage is the interaction of the powerful WR wind with the circumstellar medium.

From the above considerations, a WR ring nebula can be composed of (a) swept-up interstellar gas (WBB); (b) a mixture of stellar ejecta from the LBV phase or swept-up RSG wind and swept-up interstellar gas; or (c) pure stellar ejecta if the bulk ejection of material dominates. Likewise, an LBV nebula is expected to be composed mainly of stellar material ejected in one or more violent episodes. From the second stage onwards, the nebular material will be chemically enriched, and thus abundance studies offer the possibility of providing information on the past history of nucleosynthesis and the nature of the progenitor star.

\subsection{Abundance studies}

In a series of papers, Esteban et al. (1990-1993) have determined abundances for twelve Galactic WR nebulae. The oxygen abundance, N/O ratio and the helium mass fraction (Y) of these nebulae are given in Table I. Of the sample, four are chemically enriched and classified as stellar ejecta (E), two are a mixture of stellar ejecta and swept-up interstellar gas (E+ISM). The remaining half have $\mathrm{H}$ II region abundances of which four are classified as wind-blown bubbles (WBB) and two as quiescent $\mathrm{H}$ II regions. The anomalous abundances determined for the ejecta-type WR nebulae can be used to probe the chemical processes operating during the evolution of the central star. As can be seen from Table I, the ejecta nebulae are enhanced in $\mathrm{N}$ and $\mathrm{He}$ but deficient in $\mathrm{O}$ with respect to Orion. By comparing these abundances with the surface abundances predicted by the evolutionary models of Maeder (1990), Esteban et al. (1992) find that they correspond to stars near the end of the RSG phase, with initial masses in the range $25-40 \mathrm{M}_{\odot}$. This suggests that the bulk of enriched material in these four WR ejecta nebulae was ejected during a short period of very high mass loss or shell ejection at, or very near to, the end of the RSG phase.

A recent study of two newly discovered WR nebulae in the LMC by Garnett et al. (1993) has shown that the shell around Br 13 (WN8) has a composition consistent with stellar ejecta, while that around the WN1 star Br 2 has normal LMC interstellar abundances.

The few abundance analyses that have been performed for LBV nebulae indicate that they have an origin consistent with stellar ejecta. For AG Car, Mitra \& Dufour (1990) and de Freitas Pacheco et al. (1992) find that the nebula is $\mathrm{N}$ enriched and $\mathrm{O}$ deficient. For the ejecta associated with $\eta \mathrm{Car}$, 
Davidson et al. (1986) find that most of the CNO has been processed to nitrogen. Abundance studies of the nebulae surrounding P Cyg (Johnson et al. 1992), HR Car and WRA 751 (Hutsemékers \& Van Drom 1991a,b) indicate high $\mathrm{N} / \mathrm{S}$ ratios which, assuming $\mathrm{S}$ has a normal abundance, suggest $\mathrm{N}$ overabundances. No oxygen lines are detected in any of these three nebulae, indicating high $\mathrm{O}$ deficiencies. Abundances for the nebulae associated with AG Car and $\eta$ Car are given in Table I and show similar N/O ratios to the WR ejecta nebulae. For all these objects, the strong $O$ deficiencies, when combined with the high nitrogen enhancements, suggest that we are directly observing material that has been processed via the $\mathrm{ON}$ cycle.

\section{TABLE I}

Abundances of Galactic WR and LBV nebulae.

\begin{tabular}{|c|c|c|c|c|c|c|c|}
\hline nebula & WR & type & $\begin{array}{c}\text { nebula } \\
\text { class }\end{array}$ & $\begin{array}{c}\log \mathrm{O} / \mathrm{H} \\
+12\end{array}$ & $\log N / O$ & $Y$ & ref. \\
\hline S 308 & 6 & WN5 & $\mathrm{E}$ & 8.03 & +0.22 & 0.45 & 1 \\
\hline NGC 6888 & 136 & WN6 & $\mathrm{E}$ & 8.14 & +0.27 & 0.43 & 2 \\
\hline M1-67 & 124 & WN8 & $\mathrm{E}$ & 7.98 & +0.47 & $0.47:$ & 3 \\
\hline RCW 58 & 40 & WN8 & $\mathrm{E}$ & 8.72 & -0.30 & 0.48 & 4 \\
\hline G $2.4+1.4$ & 102 & W01 & $\mathrm{E}+\mathrm{ISM}$ & 8.45: & -0.49 & 0.47 & 1 \\
\hline RCW 104 & 75 & WN6 & $\mathrm{E}+\mathrm{ISM}$ & 8.48 & -0.13 & 0.31 & 1 \\
\hline Anon & 23 & WC6 & WBB & 8.69 & -1.09 & - & 1 \\
\hline Anon & 134 & WN6 & WBB & 8.83 & -0.85 & 0.29 & 1 \\
\hline L $69.8+1.74$ & 131 & WN7 & H II & 8.78 & -1.13 & 0.28 & 1 \\
\hline NGC 2359 & 7 & WN4 & WBB & 8.24 & -0.95 & 0.30 & 5 \\
\hline NGC 3199 & 18 & WN5 & WBB & 8.47 & -0.83 & 0.32 & 1 \\
\hline RCW 78 & 55 & WN8 & H II & 8.92 & -0.85 & $\geq 0.31$ & 5 \\
\hline AG Car & & LBV & E & 7.20 & +0.30 & & 6 \\
\hline$\eta$ Car & & LBV & E & $\leq 7.80$ & $\geq 1.20$ & 0.40 & 7 \\
\hline Orion & & & H II & 8.60 & -1.00 & 0.28 & 8 \\
\hline
\end{tabular}

1. Esteban et al. (1992), 2. Esteban \& Vílchez (1992), 3. Esteban et al. (1991), 4. Rosa \& Mathis (1990), 5. Esteban et al. (1990), 6. Mitra \& Dufour (1990), 7. Davidson et al. (1986), 8. Rosa (1987). 


\subsection{Dynamics and masses of LBV nebulae}

Dynamical analyses can also provide information on the past evolutionary history of the central star as well as probing the interaction of winds and ejecta with the ISM. For AG Car, Robberto et al. (1993) used a dynamical model with the observed nebular parameters to derive the mass loss rate and wind velocity of the pre-LBV phase. They find values consistent with that of a yellow supergiant. Viotti et al. (1993) have detected a very faint $\mathrm{H}$ II halo exterior to the AG Car nebula which they suggest is the remnant wind from a previous evolutionary phase.

Recently, Hutsemékers (1994) has discovered that both the dust and ionized masses of LBV nebulae are strongly correlated with the luminosities of the central stars. He finds that this correlation constrains the formation process of the nebulae. In particular, it argues against a continuous mass loss mechanism since the nebular mass would then be a function of time. Instead, he finds good agreement between the slope of his observed nebular mass - stellar luminosity relation and that predicted by Maeder (1989), suggesting that the nebulae are formed in a single violent event through a structural instability mechanism.

\section{Nebulae as probes of stellar parameters}

Since WR nebulae appear to be photoionized, the nebulae contain information on the far-UV energy distribution of the central star. Thus by modelling the observed ionization structure, it is possible to obtain accurate stellar effective temperatures and luminosities. In applying this method, input flux distributions from non-LTE WR model atmospheres are required, and thus the shape of the models in the crucial far-UV region can be tested as well.

In a recent study (Esteban et al. 1993; Smith \& Esteban 1994), eight WR nebulae have been modelled using grids of pure helium unblanketed WR model flux distributions from Schmutz et al. $(1992,1994)$. It is found that for the hotter stars $\left(T_{*}>55000 \mathrm{~K}\right)$, the observed nebular line strengths are correctly reproduced and the derived effective temperatures are in good agreement with those of recent stellar emission line analyses (e.g., Crowther et al. 1994). This agreement suggests that the model WR flux distributions have the correct shape in the far-UV for $T_{*}>55000 \mathrm{~K}$. For the four cooler late WN stars, however, the WR model flux distributions produce too much nebular ionization and the derived effective temperatures are lower than those determined from the stellar emission lines. The reason for this discrepancy appears to be the lack of line-blanketing in the model atmospheres. Schmutz (these proceedings) discusses new line-blanketed model atmospheres for WR stars.

WR nebulae can also be used to locate very high temperature WR stars by searching for nebular He II $\lambda 4686$ emission. In the Galaxy, the WO1 star 
WR 102 is surrounded by the nebula G $2.4+1.4$ which has strong nebular He II (Dopita et al. 1990) and appears to be a mixture of stellar ejecta and interstellar gas (Esteban et al. 1992; Table I). An effective temperature of $105000 \mathrm{~K}$ is derived for the central star from a nebular analysis (Esteban et al. 1993). The WO3 star in the dwarf irregular galaxy IC 1613 is also a source of nebular He II emission. Kingsburgh \& Barlow (1994) present a detailed study of this object and nebula; they derive an effective temperature of $75000-90000 \mathrm{~K}$ from a Zanstra analysis. Regarding WN stars, He II emission is detected in the nebulae associated with the Magellanic Cloud WN1 stars Br 2 and AB7 (Pakull 1991) and the WN3+O binary Br40a (Niemela et al. 1991).

\section{Nebulae as probes of wind asymmetries}

Another area of research where nebular studies can make an important contribution is in linking morphological asymmetries in the nebulae to wind asymmetries. Coronagraphic observations of the AG Car nebula (Paresce \& Nota 1989; Nota et al. 1992) reveal that the nebular dust is contained in a bipolar structure, roughly perpendicular to the major axis of the ellipticallyshaped gaseous nebula. Spectropolarimetric observations of the wind of AG Car indicate that it is strongly asymmetric in a direction perpendicular to the nebular bipolar structure (Schulte-Ladbeck et al. 1993a). Clampin et al. (1993) have obtained coronagraphic observations of the LMC LBV R127 and find that the nebula has bipolar lobes. Again, spectropolarimetry (SchulteLadbeck et al. 1993b) reveals a highly asymmetric wind in the direction perpendicular to the two bright nebular lobes. Thus for both of these LBVs, it appears that the nebular morphology echoes the wind asymmetry, suggesting a long-lived phenomenon. HST images of $\eta$ Car and the Homunculus nebula (Hester et al. 1991) show that a stellar jet is present and that the nebula is a thin, clumpy shell. New observations of the nebula associated with P Cygni (Barlow et al. 1994) reveal that there is an inner, nearly circular nebula with an expansion age of $900 \mathrm{yr}$, and faint outer shells from earlier episodes of mass ejection.

Regarding WR nebulae, Nota et al. (these proceedings) present new results on the morphology and dynamics of the inner region of the M1-67 ejecta nebula. They find that the nebula has a bipolar structure with the expansion axis aligned with the two cones visible in the images.

\section{The Connection between LBV and WR Nebulae}

Considering that the LBV phase is immediately prior to the WR phase for stars with initial masses $>50 \mathrm{M}_{\odot}$, it seems likely that some LBV nebulae are the progenitors of WR nebulae. On abundance grounds, this seems possible 
since they share $\mathrm{N}$ enhancements and $\mathrm{O}$ deficiencies (Sect. 2.2) although strictly comparable abundance analyses have yet to be performed. Miller \& Chu (1993) find that the emission measure of ejecta nebulae is too low for detection after $\sim$ few $\times 10^{4} \mathrm{yr}$ which corresponds to $\sim 10 \%$ of the WR lifetime. Thus is it possible that the LBV ejecta have become too faint to detect around some WR stars. It is interesting to note that WN8 stars (which still have some atmospheric hydrogen) appear to be associated with ejecta nebulae (e.g., M1-67 and RCW 58 in Table I; Br 13, Garnett et al. 1993). Smith et al. (1994) have recently analysed the LBV candidate He 3-519 and AG Car at minimum. They find that both stars can be classified as WN11 stars, and that they have low $\mathrm{H} / \mathrm{He}$ ratios indicative of late $\mathrm{WN}$ stars. They therefore suggest that both stars have already evolved to an 'unstable' WN stage, and thus their nebulae can be considered as examples of WR ejecta nebulae.

Regarding future work, the LBV nebulae are less well studied than the WR nebulae; dynamical and abundance analyses would clearly be worthwhile for comparison with WR nebulae, and further investigations of nebular/wind asymmetries. It would also be interesting to search for faint halos around both types of nebulae since they provide a unique insight into the remnant winds of the progenitor stars.

\section{References}

Barlow, M.J., Drew, J.E., Meaburn, J., Massey, R.M. 1994, MNRAS 268, L29

Chiosi, C., Maeder, A. 1986, Ann. Rev. $A \& A$ 24, 329

Chu, Y.-H. 1981, ApJ 249, 195

Chu, Y.-H. 1991, in: K.A. van der Hucht \& B. Hidayat (eds.), Wolf-Rayet Stars and Interrelations with other Massive Stars in Galaxies, Proc. IAU Symp. No. 143 (Dordrecht: Kluwer), p. 349

Chu, Y.-H., Treffers, R.R., Kwitter, K.B. 1983, ApJ Suppl. 53, 937

Clampin, M., Nota, A., Golimowski, D.A., Leitherer, C., Durrance, S.T. 1993, ApJ (Letters) 410, L35

Crowther, P.A., Smith, L.J., Hillier, D.J. 1994, in: D. Vanbeveren, W. Van Rensbergen \& C. de Loore (eds.), Evolution of Massive Stars: A Confrontation between Theory and Observations, Sp. Sci. Rev. 66, 271

Davidson, K., Dufour, R.J., Walborn, N.R., Gull, T.R. 1986, ApJ 305, 867

Dopita, M.A., Lozinskaya, T.A., McGregor, P.J., Rawlings, S.J. 1990, ApJ 351, 563

Dopita, M.A., Bell, J.F., Chu, Y.-H., Lozinskaya, T.A. 1994, ApJ Suppl. in press

Drissen, L., Shara, M.M., Moffat, A.F.J. 1991, AJ 101, 1659

Esteban, C., Vílchez, J.M. 1992, ApJ 390, 536

Esteban, C., Vílchez, J.M., Manchado, A., Edmunds, M.G. 1990, $A \& A$ 227, 515

Esteban, C., Vílchez, J.M., Smith, L.J., Manchado, A. 1991, $A \mathscr{E} A$ 244, 205

Esteban, C., Vílchez, J.M., Smith, L.J., Clegg, R.E.S. 1992, $A \in A$ 259, 629

Esteban, C., Smith, L.J., Vílchez, J.M., Clegg, R.E.S. 1993, $A \& A$ 272, 299

Esteban, C., Vílchez, J.M., Smith, L.J. 1994, $A J$ 107, 1041

de Freitas Pacheco, Damineli Neto, A., Costa, R.D.D., Viotti, R. 1992, A\&A 266, 360

Garnett, D.R., Chu, Y.-H., Dopita, M.A. 1993, Rev. Mexicana Astron. Astrof. 27, 141

Heckathorn, J.N., Bruhweiler, F.C., Gull, T.R. 1982, ApJ 252, 230 
Hester, J.J., Light, R.M., Westphal, J.A., Currie, D.G., Groth, E.J., Holtzman, J.A., Lauer, T.R., O'Neil, E.J. 1991, AJ 102, 654

Hutsemékers, D. 1994, $A \mathscr{E A}$ (Letters) 281, L81

Hutsemékers, D., Van Drom, E. 1991a, $A \mathscr{B} A$ 248, 141

Hutsemékers, D., Van Drom, E. 1991b, $A \& A 251,620$

Johnson, D.R.H., Barlow, M.J., Drew, J.E., Brinks, E. 1992, MNRAS 255, 261

Kingsburgh, R.L., Barlow, M.J. 1994, $A \mathscr{E} A$ submitted

Maeder, A.F.J. 1989, in: K. Davidson, A.F.J. Moffat \&, H.J.G.L.M. Lamers (eds.), Physics of Luminous Blue Variables, Proc. IAU Coll. No. 113 (Dordrecht: Kluwer), p. 15

Maeder, A. 1990, A\&A Suppl. 84, 139

Marston, A.P., Chu, Y.-H., Garcia-Segura, G. 1994a, ApJ Suppl. in press

Marston, A.P., Yocum, D.R., Garcia-Segura, G., Chu, Y.-H 1994b, ApJ Suppl. in press

Miller, G.J., Chu, Y.-H. 1993, ApJ Suppl. 85, 137

Mitra, P.M., Dufour, R.J. 1990, MNRAS 242, 98

Niemela, V.S., Heathcote, S.R., Weller., W.G. 1991, in: K.A. van der Hucht \& B. Hidayat (eds.), Wolf-Rayet Stars and Interrelations with other Massive Stars in Galaxies, Proc. IA U Symp. No. 143 (Dordrecht: Kluwer), p. 425.

Nota, A., Leitherer, C., Clampin, M., Greenfield, P., Golimowski, D.A. 1992, ApJ 398, 621

Paresce, F., Nota, A. 1989, ApJ (Letters) 341, L83

Pakull, M.W. 1991, in: K.A. van der Hucht \& B. Hidayat (eds.), Wolf-Rayet Stars and Interrelations with other Massive Stars in Galaxies, Proc. IAU Symp. No. 143 (Dordrecht: Kluwer), p. 391.

Robberto, M., Ferrari, A., Nota, A., Paresce, F. 1993, $A \& A$ 269, 330

Rosa, M.R. 1987, in: I. Appenzeller \& C. Jordan (eds.), Circumstellar Matter, Proc. IAU Symp. No. 122 (Dordrecht: Kluwer), p. 457

Rosa, M.R., Mathis, J.S. 1990, in: C.D.Garmany (ed.), Properties of Hot Luminous Stars, ASP Conf. Series 7, 135

Schmutz, W., Leitherer, C., Grünwald, R. 1992, PASP 104, 1164

Schmutz, W., Vogel, M., Hamann, W.-R., Wessolowski, U. 1994, in preparation

Schulte-Ladbeck, R.E., Clayton, G.C., Meade, M.R. 1993a, in: J.P. Cassinelli \& E.B. Churchwell (eds.), Massive Stars: Their Lives in the Interstellar Medium, ASP Conf. Series 35, 237

Schulte-Ladbeck, R.E., Leitherer, C., Clayton, G.C., Robert, C., Meade, M.R. 1993b, ApJ 407, 723

Smith, L.J., Esteban, C. 1994, in: D. Vanbeveren, W. Van Rensbergen \& C. de Loore (eds.), Evolution of Massive Stars: A Confrontation between Theory and Observations, $S p$. Sci. Rev. 66, 263

Smith, L.J., Crowther, P.A., Prinja, R.K. 1994, $A \& A$ 281, 833

Viotti, R., Polcaro, V.F., Rossi, C. 1993, $A \& A$ 276, 432 


\section{DISCUSSION:}

Maeder: The question of the relation between LBV and WR stars is most interesting. In view of your results, the evolution could not only be O-Of-LBV-WN..., but also O-Of-WNL (H rich)LBV-WNL (H poor)...WNE as also suggested by models by Langer and ours. What is your opinion on this possibility?

Linda Smith: The problem with this scenario is that WN7 stars are not associated with ejecta nebulae unlike WN8 stars. I therefore think that most WN8 stars are probably post-LBV objects and that evolution for the most massive stars proceeds $\mathrm{O} \rightarrow \mathrm{Of} \rightarrow \mathrm{WN7}+\mathrm{abs} \rightarrow \mathrm{WN7}$ without the need for an LBV phase (see Crowther, Smith \& Hillier, these proc.).

Nussbaumer: You mentioned that you did a temperature determination with the Zanstra method. Did you assume that the nebulae are radiation bounded. If not, how did you account for the escaping radiation?

Linda Smith: The temperatures that I referred to for eight galactic WR nebulae were not derived using the Zanstra method. We used a photoionization model with a physical model describing the parameters of each WR nebula. We found that all but one of our sample of eight were density-bounded.

Conti: Can you comment on the role of line blanketing in $\mathrm{T}_{\text {eff }}$ inferred for WNL and WNE stars? Isn't it important in both cases?

Schmutz: Line blanketing is important for both types. The fact that the nebular analyses show disagreement with the analyses of the stellar spectra for WNL types but not for WNE types tells us that the effective temperatures from the spectral analyses are more or less correct for WNL types, but that their far UV flux should be blanketed. It also implies that the spectral analyses yield probably too low effective temperatures for the WNE stars since an unblanketed flux in the far UV is larger than a blanketed one. Whether a revised temperature leads to a higher temperature is however not clear because the temperature increase could be compensated by a smaller radius.

Israelian: 1. Do you have any information about of the nebulae around $B[e]$ stars?

2. Did you find any evidence of the material ejected from P Cyg in 1600 ?

Linda Smith: 1. No, I have not studied any nebulae associated with $B[e]$ stars although abundance studies would be very worthwhile for comparison with LBV nebulae.

2. The new results I described for the P Cygni nebula are the work of Barlow et al. (MNRAS in press). They note in their paper that their data hint at the possible presence of an inner nebula with a diameter of 6 arcsec which, if confirmed, could date back to circa $1600 \mathrm{AD}$.

Magalhaes: Could you comment on the evolutionary status of the $B[e]$ stars within the LBVWR nebulae picture you described?

Linda Smith: It is not clear how the B[e] stars fit into the overall evolutionary scheme of massive stars. Spectral similarities have been noted between $B[e]$ stars and some LBVs (see review of Hillier, 1992, in: The Atmospheres of Early-Type Stars, Springer, Berlin, p.105).

Nota: This is just a comment on the P Cygni nebula. We have observed it with the cororagraph and find that the nebula is mainly constituted of blobs, distributed inside a circularly symmetrical envelope. We do not see any shell, but can clearly resolve the blobs. This seems to be in agreement with the polarimetric studies by Taylor et al.

Becker: For the past 8 years we have been monitoring P Cygni with the VLA. The radio data seem to indicate an extension out to 1 arcmin, perhaps related to the optical ring around $\mathrm{P}$ Cygni. 
Polcaro: A short comment about the possibility of a mass ejection episode of P Cyg 900 years ago; I don't think that a luminosity variation of this star similar to the one of $\eta$ Car in the past century could be unnoticed from the XI Century astronomers and we have no trace of it in the historical records.

Kholtygin: What is the temperature of the dust in the nebula around AG Car? It must be rather cold, I suppose.

Linda Smith: From IRAS observations of dust in the AG Car nebula, we derive a grain temperature of 82K (see Smith, Crowther \& Prinja, 1994, A\&A 281, 833).

White: I believe that the "ladder" structures seen in HST images taken before the repair mission have not been confirmed by new images. I do not know whether the new images are in identical colours, but I think the "ladder" may not be real.

Moffat: If we all agree that WR stars are mostly He-rich, then why don't we see core temperatures that correspond to the main sequence $\left(\approx 10^{5} \mathrm{~K}\right)$ for all WR stars?

Hillier: The nebular analysis provides primarily a constraint on the extreme UV fluxes. A stellar model is still required to turn those fluxes into an effective temperature, since the models (and the nebular analysis) show that the energy curve cannot be approximated by a black body curve. The nebular models provide an important test of theoretical WR energy distributions, but cannot, in themselves, provide a stellar atmosphere model.

It is also worth emphasising that the temperatures of $>10^{5}$ predicted for a He star ignore the influence of the wind.

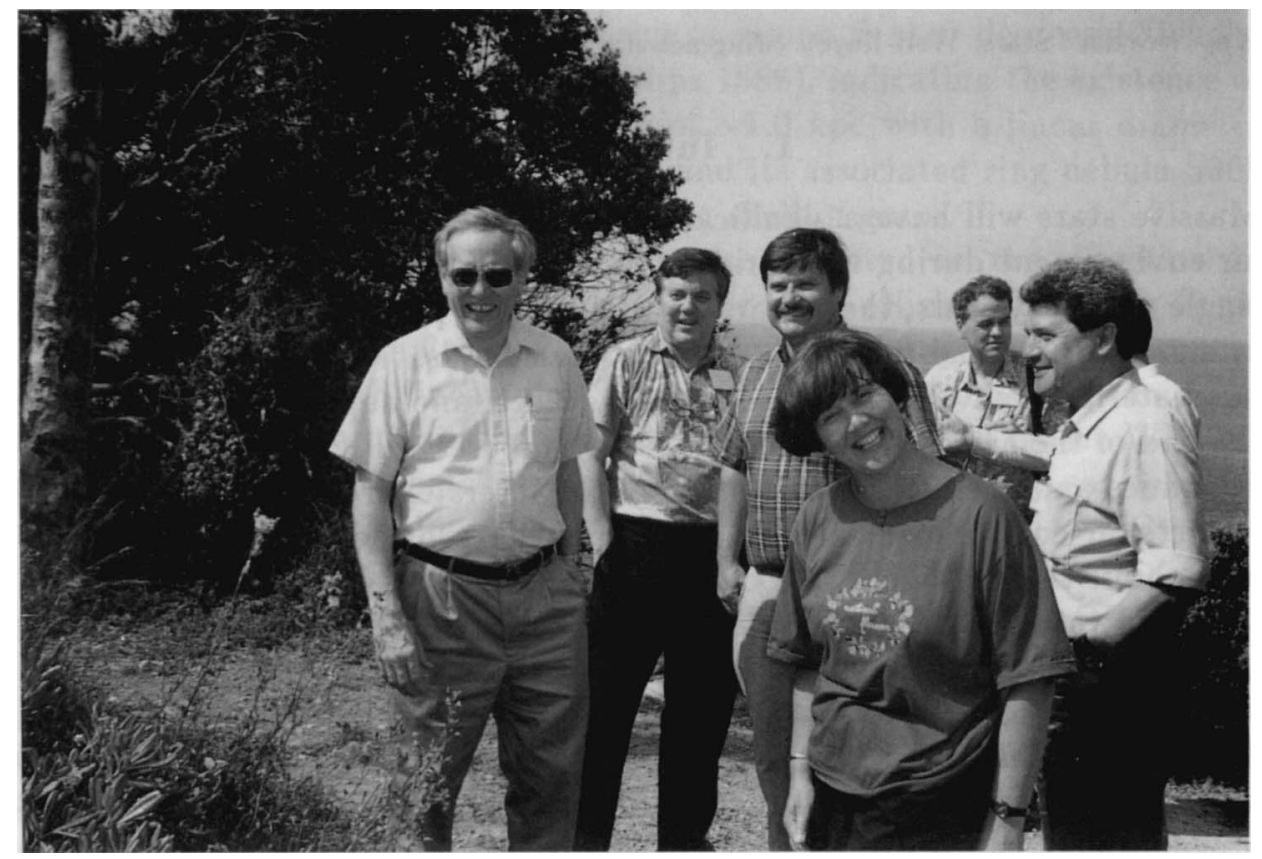

Cassinelli, Williams, Owocki, Riëtte, Moffat, Cherepashchuk 